\title{
AUTOPERCEPÇÃO DE SAÚDE EM IDOSOS COM DIABETES MELLITUS
}

\author{
Jéssica da Silva Pinheiro ${ }^{1}$, Karine Demartini ${ }^{2}$, Márcia de Oliveira Siqueira ${ }^{2}$, Andréa \\ Bona Ughini ${ }^{2}$, Matheus Santos Gomes Jorge ${ }^{2}$ e Lia Mara Wibelinger ${ }^{2}$ \\ ${ }^{1}$ UNIJUÍ*, ${ }^{2}$ UPF \\ *jessica.pinheiro88@hotmail.com
}

Acesso DOI: http://dx.doi.org/10.34059/ciejop.2019v27i1-7

\section{Resumo}

Introdução: O diabetes mellitus é uma doença metabólica que pode causar inúmeros efeitos adversos a saúde física e psicossocial dos indivíduos acometidos. Comumente observada em idosos, é necessário compreender como esta população autopercebe sua saúde. Objetivo: verificar a autopercepção de saúde em idosos com diabetes mellitus. Metodologia: Estudo observacional descritivo, que avaliou 351 idosos, de ambos os sexos, residentes na zona urbana do município de Passo Fundo/RS. Os indivíduos responderam a um questionário estruturado, cujo mesmo abordou dados sobre o perfil sociodemográfico e o perfil de saúde dos entrevistados. Os critérios de inclusão foram o diagnóstico de diabetes mellitus, residir na zona urbana do município de Passo Fundo/RS, capacidade de comunicar-se verbalmente com o entrevistador e que concordaram em assinar o Termo de Consentimento Livre e Esclarecido. Os critérios de exclusão foram indivíduos com acometimento auditivo ou disartria, residentes em instituições de longa permanência ou hospitalizados. Resultados: Dos 351 inicialmente recrutados, apenas 54 foram selecionados. A maioria era do sexo feminino, tinha entre 60-69 anos e tinha baixo nível de escolaridade, era tabagista e etilista, sofreu quedas ou hospitalizações no último ano, não tinha dificuldades para realizar as atividades de vida diária, tinha condições de saúde visual ruins, realizava fisioterapia e era praticante de atividade física. A maioria da amostra referiu perceber sua saúde como regular. Conclusão: Idosos com diabetes mellitus autopercebem sua saúde como regular.

Palavras-chave: Diabetes Mellitus; Idoso; Perfil de Saúde; Geriatria

Abstract
Introduction: Diabetes mellitus is a metabolic disease that can cause numerous adverse effects to the physical and psychosocial health of the individuals affected. Commonly observed in the elderly, it is necessary to understand how this population self-perceives their health. Objective: to verify the self-perception of health in the elderly with diabetes mellitus. Methodology: Descriptive observational study, which evaluated 351 elderly, of both sexes, living in the urban area of Passo Fundo/RS. Individuals answered a structured questionnaire, which included data on the sociodemographic profile and the health profile of the interviewees. The inclusion criteria were the diagnosis of diabetes mellitus, residents of the urban area of the municipality of Passo Fundo/RS, ability to communicate verbally with the interviewer and who agreed to sign the Informed Consent Term. Exclusion criteria were individuals with auditory impairment or dysarthria, living in long-term institutions or hospitalized. Results: Of the 351 initially recruited, only 54 were selected. The majority were female, between 60-69 years old and had low level of education, were 
smokers and alcoholics, suffered falls or hospitalizations in the last year, did not had difficulty in the activities of daily living, had poor visual health conditions, performed physical therapy and were practiced physical activity. Most of the sample referred to perceive their health as regular. Conclusion: Elderly with diabetes mellitus selfperceive their health as regular.

Keywords: Diabetes Mellitus; Aged; Health Profile; Geriatrics

\section{Introdução}

O envelhecimento populacional é um fenômeno mundial resultante do declínio da fecundidade e dos diversos avanços tecnológicos e da medicina. $O$ crescimento do número de idosos reflete em um grande desafio frente às condições de vida adequadas (Pereira et al., 2011).

O conceito de saúde está vinculado aos aspectos físicos, políticos, sociais, econômicos e culturais do indivíduo (Buss, 2000). Assim, envelhecer torna este conceito de saúde amplamente variável, pois a autopercepção de saúde está concatenada à proatividade, a autoestima, a emancipação e a relação do indivíduo com a sociedade (Borges et al., 2014; Silva et al., 2014). A autopercepção de saúde é um inquérito subjetivo sobre como o sujeito percebe a sua própria, e este tema tem sido estudado em pesquisas epidemiológicas. Além disso, é um indicativo da qualidade de vida que precede a queda das funções fisiológicas e a mortalidade (Aguilar-Palacio et al., 2015).

Com o aumento da expectativa de vida dos brasileiros, ocorre também um aumento de doenças crônicas (Presta et al., 2011), dentre as quais pode-se citar o Diabetes Mellitus (DM), uma patologia cuja etiologia desencadeia mudanças no contexto social e impacto na qualidade de vida do indivíduo, exigindo mudanças no estilo de vida. Estima-se que, no mundo, 387 milhões sejam acometidas por DM, com projeções de 471 milhões para 2035 (Federação Internacional de Diabetes, 2014). No Brasil, a prevalência de DM em indivíduo com idade entre 30 e 69 anos é de $7,6 \%$, aumentando para $20 \%$ após os 70 anos, sendo que até $50 \%$ desconhecem o diagnóstico e $25 \%$ não realize o tratamento recomendado (Sociedade Brasileira de Diabetes, 2016).

O DM é uma doença altamente limitante que pode causar danos, disfunção e falência de vários órgãos, principalmente rins, olhos, nervos, coração e vasos sanguíneos, a longo prazo. Os indivíduos acometidos pelo DM têm maior risco de apresentar associação com outras doenças como hipertensão arterial, doença coronariana, doença arterial periférica e doença vascular cerebral. Além disso, Fórum Internacional de Qualidade de Vida e Saúde - Curitiba, 15 de junho de 2019 
podem apresentar neuropatia, artropatia e disfunção autonômica, especialmente os idosos (Silva et al., 2006). Tais consequências podem resultar em impacto da saúde desta população. Em virtude disso, o objetivo deste estudo foi verificar a autopercepção de saúde em idosos com DM.

\section{Metodologia}

Estudo quantitativo, descritivo de corte transversal, cujo mesmo faz parte de um projeto denominado "Caracterização das condições de saúde dos idosos do município de Passo Fundo/RS", aprovado pelo Comitê de Ética em Pesquisa em Seres Humanos da Universidade de Passo Fundo, sob o parecer $n^{\circ}$ 447/2010 e que está em consonância com a resolução 196/96 do Conselho Nacional de Saúde.

Avaliaram-se 351 idosos por meio de uma amostragem aleatória simples. Os critérios de inclusão definidos foram para idosos de ambos os sexos, autorrelato de DM, residentes da zona urbana do município de Passo Fundo/RS, com capacidade de comunicação verbal e cognitiva para com o entrevistador e que concordaram em assinar o Termo de Consentimento Livre e Esclarecido. Os critérios de exclusão definidos foram para indivíduos com acometimento auditivo ou disartria, residentes em instituições de longa permanência ou hospitalizados.

Os participantes responderam a um questionário estruturado que foi elaborado e adaptado pelos próprios pesquisadores. Analisaram-se os itens referentes à caracterização sociodemográfica (gênero, faixa etária e escolaridade) e as condições de saúde (tabagismo, etilismo, ocorrência de quedas, dificuldade nas atividades de vida diária, condições visuais, internação hospitalar, se realiza/realizou fisioterapia, se é praticante de atividade física e a autopercepção de saúde).

A coleta de dados foi realizada, entre agosto de 2011 e setembro de 2012, por graduandos do curso de Fisioterapia da Universidade de Passo Fundo, em locais públicos da zona urbana do município de Passo Fundo/RS. Ao fim, as informações foram tabuladas em um software estatístico e realizada a análise estatística descritiva.

\section{Resultados}

Dos 351 idosos inicialmente entrevistados, apenas 54 foram identificados com diabetes mellitus (15,38\% da amostra inicial). Todos estes indivíduos preencheram Fórum Internacional de Qualidade de Vida e Saúde - Curitiba, 15 de junho de 2019 
os critérios de inclusão do presente estudo e tiveram seus dados analisados. A Tabela 1 apresenta a caracterização sociodemográfica dos idosos com diabetes mellitus.

Tabela 1. Caracterização sociodemográfica dos idosos com diabetes mellitus. Passo Fundo/RS, 2019

Variáveis N (\%)

Sexo

Feminino

$34(63,0)$

Masculino

$20(37,0)$

Faixa etária

60-69 anos

70-79 anos

$20(37,0)$

80-89 anos

$07(13,0)$

90 anos ou mais

$01(1,9)$

Escolaridade

Ensino fundamental incompleto ou completo

$40(74,0)$

Ensino médio incompleto ou completo

$11(20,4)$

Ensino superior incompleto ou completo

$03(5,6)$

Legenda: N (valor absoluto); \% (valor relativo)

O perfil sociodemográficos dos idosos com DM, demonstrou que a maioria da amostra é composta por mulheres, não longevas (menos de 80 anos de idade) e com escolaridade a nível de ensino fundamental. A Tabela 2 apresenta as condições de saúde dos idosos com diabetes mellitus. 
Tabela 2. Condições de saúde dos idosos com diabetes mellitus. Passo Fundo/RS, 2019

Variáveis $\mathrm{N}(\%)$

Tabagismo

$\operatorname{Sim}$

$42(77,8)$

Não

$12(22,2)$

Etilismo

Sim

$40(74,1)$

Não

$14(25,9)$

Quedas

Sim

$32(59,3)$

Não

$22(40,7)$

Dificuldades para realizar atividades de vida diárias

Sim

$18(33,3)$

Não

$36(66,7)$

Alteração visual

Sim

$43(79,6)$

Não

$11(20,4)$

Qualidade visual

Ruim

$10(18,5)$

Regular

$33(61,1)$

Boa

$11(20,4)$

Uso de dispositivo para correção

Sim

$37(68,5)$

Não

$17(31,5)$

Internação hospitalar no último ano

Sim

$28(51,9)$

Não

$26(48,1)$

Fisioterapia 
Atividade física

Sim

Legenda: N (valor absoluto); \% (valor relativo)

A maioria dos idosos com DM eram tabagistas e etilistas, sofreram quedas no último ano, porém não tinham dificuldades para realizar suas atividades de vida diária. Tinham alterações visuais, consideravam a qualidade da sua visão regular e faziam uso de dispositivo para correção da visão. Ainda, a maior parte da amostra sofreu internações hospitalares no último ano, realizava fisioterapia e era praticante de atividade física. A Tabela 3 apresenta a autopercepção de saúde dos idosos com diabetes mellitus.

Tabela 3. Autopercepção de saúde dos idosos com diabetes mellitus. Passo Fundo/RS, 2019

Autopercepção de saúde $\quad$ N (\%)

Péssima

Ruim

Regular

Boa

Ótima
$01(1,9)$

$03(5,6)$

$27(50,0)$

$22(40,7)$

$01(1,9)$

Legenda: N (valor absoluto); \% (valor relativo)

A maioria dos idosos com DM da amostra estudada referiram perceber sua saúde como regular.

Discussão

Idosos com DM apresentam maior risco de morte prematura e maior índice de outras comorbidades associadas, em especial as grandes síndromes geriátricas. 
Desta forma, esta população é mais suscetível a apresentar prejuízos na capacidade funcional, autonomia e pior qualidade de vida, impactando o sistema de saúde, a família e o próprio idoso acometido (Sociedade Brasileira de Diabetes, 2016; Francisco et al., 2010). O que justifica a necessidade da realização do presente estudo, onde observamos que os idosos com DM eram na sua maioria mulheres, não longevos e com baixo nível de escolaridade. Além disso, a maioria da amostra era tabagista e etilista, sofreu quedas ou hospitalizações no último ano, não tinha dificuldades para realizar as atividades de vida diária, tinha condições de saúde visual ruins, realizava fisioterapia, era praticante de atividade física e autopercebia sua saúde como regular.

O perfil sociodemográfico da nossa amostra apontou para predominância do sexo feminino, da faixa etária dos 60-69 anos e com nível escolar fundamental completo ou incompleto, dados estes que são confirmados de acordo com a literatura, tanto em relação ao sexo (Melo-Silva et al., 2018; Batista et al., 2011), a idade (Instituto Brasileiro De Geografia e Estatística, 2018) e a escolaridade (Instituto Brasileiro De Geografia e Estatística, 2014; Kummer et al, 2014).

Nossos resultados que apontaram que a maioria da amostra com DM era composta por mulheres, concorda com outro estudo onde identificou-se que em uma amostra de 1.515 indivíduos portadores da doença a maior prevalência foi do sexo feminino (63\%). Além disso, a média de idade dos participantes eram de 65 anos (Rossaneis et al., 2016). Ainda, outro estudo realizado com 196 idosos com DM documenta que a maioria da amostra eram analfabetos ou com poucos anos de estudo (Lima et al., 2016). Tais dados corroboram com nossos achados sociodemográficos a respeito do perfil dos idosos com DM, onde a maior prevalência foi de mulheres, na faixa etária dos 60-69 anos e com escolaridade a nível de ensino fundamental.

Uma pesquisa realizada com 196 idosos com DM, demonstrou um baixo índice de tabagismo $(13,3 \%)$ e etilismo $(9,2 \%)$ (Lima et al., 2016). Outro estudo demonstrou que idosos que não faziam uso de álcool apresentaram maior prevalência de DM (Mendes et al., 2011). Tais informações, vão de encontro aos dados por nós apresentados, onde observamos uma elevada prevalência de tabagismo e etilismo nos idosos com DM, cujos mesmos autoperceberam sua saúde como regular. 
Nossos dados referentes às condições visuais mostram prevalência de alteração visual, e um expressivo número de indivíduos que fazem uso de dispositivos de correção. Quando perguntados sobre a qualidade da visão, a maioria classifica-a com regular. Em um estudo realizado com idosos com DM em uma unidade básica de saúde demonstrou que $74,5 \%$ da amostra apresentou algum tipo de alteração visual (Lima et al., 2016), corroborando com nossos achados.

Nos idosos de nossa pesquisa houve uma grande prevalência de quedas e um terço dos entrevistados relataram dificuldades nas atividades de vida diária. Um estudo epidemiológico conduzido com 38.200 idosos chineses demonstrou que as quedas são uma das cinco causas mais comuns de morte (Meng et al., 2019). Entre os idoso com DM a prevalência de quedas permeia entre os $25 \%$ e naqueles sem a doença em torno de $18,2 \%$, evidenciando que o DM é uma condição que apresenta um alto risco para quedas (Yang et al, 2016).

Idosos com DM apresentam maiores índices de internação hospitalar no último ano (Mendes et al., 2011) e o aumento no número de internações que tem ocorrido nos últimos 12 anos é preocupante, visto que esse fenômeno é associado à deterioração das habilidades motoras e a redução da qualidade de vida (Covino et al., 2019). O que concorda com nosso estudo onde observamos que mais da metade da amostra sofreu internações hospitalares no último ano. Por outro lado, algumas fontes se contrapõem aos nossos achados. Um estudo que avaliou 9.389 idosos verificou que a prevalência de hospitalizações no último ano foi de $10,29 \%$, embora o DM uma das quatro principais causas (Melo-Silva et al, 2018), o que corrobora com outro estudo, que foi realizado em Campinas, São Paulo, onde demonstrou-se que apenas $16,6 \%$ dos idosos com DM foram internados no último ano. (Prado et al, 2016).

Quando questionados sobre realizarem tratamento fisioterapêutico ou praticarem algum tipo de atividade física, $53,7 \%$ e $75,9 \%$ responderam afirmativamente para estas questões, respectivamente. A fisioterapia no DM é uma abordagem benéfica, podendo ser de caráter reabilitativo, atuando sobre as complicações neurológicas, osteomusculares e vasculares, quanto de caráter preventivo, incentivando a ações preventivas primária ou secundárias (Portes, 2015). Neste sentido, pode-se compreender o porquê de mais de metade da amostra realizar algum tipo de tratamento fisioterapêutico. Além disso, acreditamos que o elevado número de idosos com DM serem praticantes de algum tipo de Fórum Internacional de Qualidade de Vida e Saúde - Curitiba, 15 de junho de 2019 
atividade física possa estar relacionado ao fato de que muitos idosos procuram a atividade física com caráter curativo aos problemas de saúde já instalados e não como forma de prevenção (Gomes e Zazá, 2009). Todavia, nossos resultados sobre este aspecto divergem dos achados apresentados em outro estudo, onde o número de idosos praticantes de atividade física foi de 42,9\% (Lima et al., 2016).

A autopercepção de saúde refere-se a percepção que o indivíduo possui sobre sua própria saúde, um importante indicador de promoção de saúde, bem-estar e qualidade de vida que pode influenciar na quantidade de utilização de serviços de saúde. Ainda, a autopercepção de saúde é individual e é determinada pelos traços da personalidade, do estado de humor e das experiências físicas prévias do indivíduo (Bigaton et al., 2015; Schneider et al, 2004). Razão esta pela qual estudamos a autopercepção de saúde dos idosos com DM.

Nossos achados apontaram que a maioria dos idosos com DM referiram sua saúde como regular. Dados da Pesquisa Nacional de Saúde de 2013 apontam que $66,1 \%$ dos brasileiros acima dos 18 anos perceberam sua saúde como boa ou muito boa, sendo que com o avanço da idade esses números tendem a declinar, podendo atingir menos de 50\% (48,4\%) nos idosos (Instituto Brasileiro De Geografia e Estatística, 2014). Este achado é reforçado por uma pesquisa na região Sul do Brasil, realizada com 301 idosos, onde 47,5\% referiram seu estado de saúde como regular (Rigo et al, 2017). Ao levantar estes dados em idosos portadores de DM observa-se que até $74,3 \%$ pode perceber sua como regular ou ruim (Corralo et al., 2018), corroborando com os resultados da presente pesquisa.

Em um estudo realizado com idosos com DM demonstrou que a maioria da amostra apresentava uma autopercepção de saúde ruim que foi identificada como fator associado à doença (Vitoi et al., 2015), bem como outro estudo que verificou que a autopercepção de saúde negativa (ruim ou muito ruim) foi um fator associado ao DM (Mendes et al., 2011). Embora, muitas vezes, a autopercepção de saúde seja classificada como negativa, como no estudo de Ramos et al. (2017) (onde não foi constatada associação entre a autopercepção de saúde e o DM), observamos que nossos dados se opõem aos estudos supracitados, pois observamos que os idosos com DM apresentaram uma autopercepção de saúde regular.

Este estudo pode apresentar limitações. O tamanho amostral que mostrou-se inferior ao tamanho das amostras de outros estudos, associado a forma de identificação do DM, que foi por meio do autorrelato, e ao fato de não ter-se Fórum Internacional de Qualidade de Vida e Saúde - Curitiba, 15 de junho de 2019 
diferenciado as doenças entre DM tipo I e DM tipo II, podem ter sido fatores limitantes do presente estudo. Embora essas limitações sejam existentes elas não impedem a generalização dos dados encontrados, incentivando-se a novos estudos longitudinais com amostras mais robustas.

\section{Conclusão}

Os idosos com DM percebem sua saúde como regular, o que pode estar ligado a maior prevalência de baixa escolaridade, tabagismo, etilismo, acidente por quedas, piores condições da visão e internações hospitalares encontrados na amostra estudada.

\section{Referências}

AGUILAR-PALACIO, Isabel; CARRERA-LASFUENTES, Patricia; RABANAQUE, Maria José. Salud percibida y nivel educativo en España: tendencias por comunidades autónomas y sexo (2001-2012). Gaceta Sanitaria, Barcelona, v. 29, n. 1, p. 37-43, 2015.

BATISTA, Juliana Secchi; PRESTA, Sara Antoniuk; VIDMAR, Marlon Francys; WIBELINGER, Lia Mara. Caracterização e condições de saúde dos idosos do município de Passo Fundo. Revista Contexto \& Saúde, ljuí, v.10, n.20, p. 799-806, 2011

BIGATON, Eduardo Siqueira; MYRA, Rafaela Simon; DE MARCO, Mariângela; JORGE, Matheus Gomes Santos; WIBELINGER, Lia Mara. Autopercepção de saúde em idosos portadores de doenças osteoarticulares praticantes de atividade física. Revista Eletrônica Acervo Saúde, Campinas, v. 7, n. 1, p. 742-747, 2015.

BORGES, Amanda Morás; SANTOS, Grasiela; KUMMER, Julia Andréia; FIOR, Laura; DAL MOLIN, Vinícius; WIBELINGER, Lia Mara. Autopercepção de saúde em idosos residentes em um município do interior do Rio Grande do Sul. Revista Brasileira de Geriatria e Gerontologia, Rio de Janeiro, v. 17, n. 1, p. 79-86, 2014.

BUSS, Paulo Marchiori. Promoção da saúde e qualidade de vida. Ciência Saúde Coletiva, Rio de Janeiro, v. 5, n.1, p. 163-177, 2000.

COVINO, Marcello; PETRUZZIELLO, Carmine; ONDER, Graziano; MIGNECO, Alessio; SIMEONI, Benedetta; FRANCESCHI, Francesco; OJETTI, Veronica. A 12year retrospective analysis of differences between elderly and oldest old patients referred to the emergency department of a large tertiary hospital. Maturitas, Amsterdam, v. 120, n. 1, p. 7-11, 2019.

CORRALO, Vanessa da Silva; BINOTTO, Vanessa Marconatto; BOHNEN, Lilian Caroline; SANTOS, Guilherme Augusto Gonzaga dos; DE-SÁ, Clodoaldo Antônio. 
Polifarmácia e fatores associados em idosos diabéticos. Revista de Salud Pública, Bogotá, v. 20, n. 3, p. 366-372, 2018.

FEDERAÇÃO INTERNACIONAL DE DIABETES. Diabetes Atlas. $6^{a}$ ed. Bruxelas: Federação Internacional de Diabetes. 2014. Disponível em: <https://www.idf.org/elibrary/epidemiology-research/diabetes-atlas.html>. Acesso em: 11 abr 2019.

FRANCISCO, Priscila Maria Stolses Bergamo; BELON, Ana Paula; BARROS, Marilisa Berti de Azevedo; CARANDINA, Luana; ALVES, Maria Cecília Goi Porto; GOLDBAUM, Moises; CESAR, Chester Luiz Galvão. Diabetes auto-referido em idosos: prevalência, fatores associados e práticas de controle. Cadernos de Saúde Pública, Rio de Janeiro, v. 26, n. 1, p. 175-184, 2010.

GOMES, Kátia Virgínia; ZAZÁ, Daniela Coelho. Motivo de adesão à prática de atividade física em idosas. Revista Brasileira de Atividade Física e Saúde, Florianópolis, v. 14, n. 2, p. 969-984, 2009.

INSTITUTO BRASILEIRO DE GEOGRAFIA E ESTATÍSTICA. Projeção da População do Brasil e das Unidades da Federação. Brasil, 2018. Disponível em: <https://www.ibge.gov.br/apps/populacao/projecao/>. Acesso em: 26 mar 2019.

INSTITUTO BRASILEIRO DE GEOGRAFIA E ESTATÍSTICA. Pesquisa Nacional de Saúde -2013. Percepção do estado de saúde, estilo de vida e doenças crônicas: Brasil, grandes regiões e unidades da federação. Brasil, 2014. Disponível em: <https://ww2.ibge.gov.br/home/estatistica/populacao/pns/2013/default.shtm>. Acesso em: 26 mar 2019.

KUMMER, Júlia Andréia; FAREZIN, Skarlet; WIBELINGER, Lia Mara. Caracterização das condições de saúde de idosos participantes de grupos de convivência. Lecturas: Educación Física y Deportes, Buenos Aires, v. 19, n. 194, p. 1-1, 2014.

LIMA, Luciano Ramos de; FUNGHETTO, Silvana Schwerz; VOLPE, Cris Renata Grou; SANTOS, Walterlânia Silva; FUNEZ, Mani Indiana; STIVAL, Marina Morato. Qualidade de vida e o tempo do diagnóstico do diabetes mellitus em idosos. Revista Brasileira de Geriatria e Gerontologia, Rio de Janeiro, v. 21, n. 2, p. 180-190, 2018.

MELO-SILVA, Alexandre Moreira de; MAMBRINI, Juliana Vaz de Melo; SOUZA JUNIOR, Paulo Roberto Borges de; ANDRADE, Fabíola Bof de; LIMA-COSTA, Maria Fernanda. Hospitalizations among older adults: results from ELSI-Brazil. Revista de Saúde Pública, São Paulo, v. 52, supl. 2, 3s, p. 1-1, 2018.

MENDES, Telma de Almeida Busch; GOLDBAUM, Moisés; SEGRI, Neuber José; BARROS, Marilisa Berti de Azevedo; CESAR, Chester Luiz Galvão; CARANDINA, Luana; ALVES, Maria Cecília Goi Porto. Diabetes mellitus: fatores associados à prevalência em idosos, medidas e práticas de controle e uso dos serviços de saúde em São Paulo, Brasil. Cadernos de Saúde Pública, Rio de Janeiro, v. 27, n. 6, p. 1233-1243, 2011. 
MENG, Ruillin; XU, Xiaojun; XU, Yanjun; LUO, Chao; XU, Haofeng; WANG, Ye; SONG, Xiuling; XIA, Liang; XIAO, Ni; ZHOU, Shaoen; LIN, Lifeng. Epidemiological characteristics of injury mortality in Guangdong Province, China, 2015. BMC Public Health, London, v. 19, n. 21, p. 2019.

PEREIRA, Keila Cristina Rausch; ALVAREZ, Angela Maria; TRAEBERT, Jefferson Luiz. Contribuição das condições sociodemográficas para a percepção da qualidade de vida em idosos. Revista Brasileira de Geriatria e Gerontologia, Rio de Janeiro, v. 14 , n. 1, p. 85-95, 2011.

PORTES, Leonardo Henriques. Abordagem do fisioterapeuta no diabetes mellitus: revisão de literatura. Arquivos de Ciências da Saúde, Campinas, v. 22, n. 3, p. 914, 2015.

PRADO, Maria Aparecida Medeiros Barros do; FRANCISCO, Priscila Maria Stolses Bergamo; BARROS, Marilisa Berti de Azevedo. Diabetes em idosos: uso de medicamentos e risco de interação medicamentosa. Ciência \& Saúde Coletiva, Rio de Janeiro, v. 21, n. 11, p. 3447-3458, 2016.

PRESTA, Sara Antoniuk; VIDMAR, Marlon Francys; BATISTA, Juliana Secchi; SILVEIRA, Michele Marinho; WIBELINGER, Lia Mara. Caracterização e condições de saúde dos idosos do município de Passo Fundo, no Rio Grande do Sul. Revista Brasileira de Ciências da Saúde, São Caetano do Sul, v. 9, n. 29, p. 31-38, 2011.

RAMOS, Roberta de Souza Pereira da Silva; MARQUES, Ana Paula de Oliveira; RAMOS, Vânia Pinheiro; BORBA, Anna Karla de Oliveira Tito; AGUIAR, Avelino Maciel Alves de; LEAL, Márcia Carréra Campos. Fatores associados ao diabetes em idosos assistidos em serviço ambulatorial especializado geronto-geriátrico. Revista Brasileira de Geriatria e Gerontologia, Rio de Janeiro, v. 20, n. 3, p. 364-374, 2017.

RIGO, Lilian; GARBIN, Raíssa Rigo; RODRIGUES, José Lucas Sani de Alcântara; MENEZES-JÚNIOR, Laerte Ribeiro; PARANHOS, Luiz Renato; BARELLI, Cristiane. Self-perceived quality of health and satisfaction by elderly seen by the Family Health Strategy team. Einstein (São Paulo), São Paulo, v. 15, n. 4, p. 428-434, 2017.

ROSSANEIS, Mariana Angela; HADDAD, Maria do Carmo Fernandez Lourenço; MATHIAS, Thaís Aidar de Freitas; MARCON, Sonia Silva. Differences in foot selfcare and lifestyle between men and women with diabetes mellitus. Revista LatinoAmericana de Enfermagem, Ribeirão Preto, v. 24, e2761, 2016.

SCHNEIDER, Gudrun; DRIESCH, Georg; KRUSE, Andreas; WACHTER, Michael; NEHEN, Hans-Georg; HEUFT, Gereon. What influences self-perception of health in the elderly? The role of objective health condition, subjective well-being and sense of coherence. Archives of Gerontology and Geriatrics, Amsterdam, v. 39, n. 3, p. 227-237, 2004.

SILVA, Terezinha Rodrigues; FELDMAM, Chaie; LIMA, Maria Helena A.; NOBREI, Moacyr R. Cuce; DOMINGUES, Rachel Z. L. Controle de diabetes mellitus e hipertensão arterial com grupos de intervenção educacional e terapêutica em 
seguimento ambulatorial de uma unidade Básica de saúde. Saúde e Sociedade, São Paulo, v. 15, n. 3, p. 180-189, 2006.

SILVA, Isnanda Tarciara da; PINTO JUNIOR, Elzo Pereira; VILELA, Alba Benemérita Alves. Autopercepção de saúde de idosos que vivem em estado de corresidência. Revista Brasileira de Geriatria e Gerontologia, Rio de Janeiro, v. 17 , n. 2, p. $275-287,2014$

SOCIEDADE BRASILEIRA DE DIABETES. Diretrizes da Sociedade Brasileira de Diabetes: 2015-2016. São Paulo: AC Farmacêutica. 2016. Disponível em: <http://www.diabetes.org.br/profissionais/images/docs/diretrizes-sbd-20152016.pdf>. Acesso em: 11 abr 2019.

VITOI, Nayla Cordeiro; FOGAL, Aline Siqueira; NASCIMENTO, Clarissa de Matos; FRANCESCHINI, Sylvia do Carmo Castro; RIBEIRO, Andréia Queiroz. Prevalência e fatores associados ao diabetes em idosos no município de Viçosa, Minas Gerais. Revista Brasileira de Epidemiologia, São Paulo, v. 18, n. 4, p. 953-965, 2015.

YANG, Yu; HU, Xinhua; ZHANG, Qiang; ZOU Rui. Diabetes mellitus and risk of falls in older adults: a systematic review and meta-analysis. Age and Ageing, London, v. 15, n. 6, p. 761-767, 2016. 